\title{
Placental polyamine metabolism differs by fetal sex, fetal growth restriction, and preeclampsia
}

\author{
Sungsam Gong, ${ }^{1}$ Ulla Sovio, ${ }^{1,2}$ Irving L.M.H. Aye, ${ }^{1,2}$ Francesca Gaccioli, ${ }^{1,2}$ Justyna Dopierala,, ${ }^{1,2}$ \\ Michelle D. Johnson, ${ }^{1,2}$ Angela M. Wood, ${ }^{3}$ Emma Cook, ${ }^{1}$ Benjamin J. Jenkins, ${ }^{4}$ Albert Koulman, ${ }^{4}$ \\ Robert A. Casero Jr., ${ }^{5}$ Miguel Constância, ${ }^{1,2,6}$ D. Stephen Charnock-Jones, ${ }^{1,2}$ and Gordon C.S. Smith ${ }^{1,2}$ \\ 'Department of Obstetrics and Gynaecology, NIHR Cambridge Biomedical Research Centre, ${ }^{2}$ Centre for Trophoblast \\ Research (CTR), Department of Physiology, Development and Neuroscience, ${ }^{3}$ Department of Public Health and Primary \\ Care, ${ }^{4}$ NIHR BRC Core Metabolomics and Lipidomics Laboratory, University of Cambridge, Cambridge, United Kingdom. \\ ${ }^{5}$ The Sidney Kimmel Comprehensive Cancer Center, Johns Hopkins University School of Medicine, Baltimore, Maryland, \\ USA. 'University of Cambridge Metabolic Research Laboratories and MRC Metabolic Diseases Unit, Wellcome Trust-MRC \\ Institute of Metabolic Science, Cambridge, United Kingdom.
}

Preeclampsia and fetal growth restriction (FCR) are major causes of the more than $\mathbf{5}$ million perinatal and infant deaths occurring globally each year, and both are associated with placental dysfunction. The risk of perinatal and infant death is greater in males, but the mechanisms are unclear. We studied data and biological samples from the Pregnancy Outcome Prediction (POP) study, a prospective cohort study that followed 4,212 women having first pregnancies from their dating ultrasound scan through delivery. We tested the hypothesis that fetal sex would be associated with altered placental function using multiomic and targeted analyses. We found that spermine synthase (SMS) escapes X-chromosome inactivation (XCI) in the placenta and is expressed at lower levels in male primary trophoblast cells, and male cells were more sensitive to polyamine depletion. The spermine metabolite N1,N12-diacetylspermine (DiAcSpm) was higher in the female placenta and in the serum of women pregnant with a female fetus. Higher maternal serum levels of DiAcSpm increased the risk of preeclampsia but decreased the risk of FCR. To our knowledge, DiAcSpm is the first maternal biomarker to demonstrate opposite associations with preeclampsia and FGR, and this is the first evidence to implicate polyamine metabolism in sexrelated differences in placentally related complications of human pregnancy.

License: This work is licensed under the Creative Commons Attribution 4.0 International License. To view a copy of this license, visit http:// creativecommons.org/licenses/ by/4.0/.

Authorship note: SG, US, ILMHA, and FG are co-first authors. DSC] and GCSS are co-senior authors.

Conflict of interest: GCSS reports equipment loans and consumable support from Roche Diagnostics and has attended an Advisory Board for Roche.

Submitted: February 26, 2018

Accepted: May 31, 2018

Published: July 12, 2018

\section{Reference information:}

JCI Insight. 2018;3(13):e120723.

https://doi.org/10.1172/jci.

insight.120723.

\section{Introduction}

Preeclampsia and FGR are 2 of the "great obstetrical syndromes" (1). These disorders cause short-term complications for the infant and have been associated with a range of diseases in adult life, such as ischemic heart disease, stroke, type 2 diabetes, and long-term neurodevelopmental disorders (2). Moreover, these complications have profound effects on maternal morbidity and mortality. Preeclampsia is one of the leading causes of maternal death globally, and both preeclampsia and FGR are markers for the mother's later risk of cardiovascular disease (3). Both preeclampsia and FGR are associated with abnormal placental function and metabolism (4). Currently, the placental mechanisms leading to preeclampsia and FGR are only partially understood. It is also unknown why placental dysfunction can lead to preeclampsia without FGR in one woman and FGR without preeclampsia in another. Epidemiological studies have demonstrated that fetal sex is associated with different patterns of ultrasonic markers of placentation (5), placental pathology (6), the risk of perinatal death (7-10), and the risk of preeclampsia $(11,12)$, but the mechanisms underlying these differences are unknown. Given these observations, we hypothesized that the placenta would exhibit sex-related differences across multiomic analyses. Moreover, we hypothesized that sex-related differences in placental function would be associated with the risk of placentally related complications of human pregnancy. We tested these hypotheses using 3 discovery-based methods, namely analysis of the placental transcriptome, the placental methylome, and the mother's serum metabolome. We then used targeted in vitro experiments in primary cultures of human trophoblast cells to confirm sex-related differences in function. 


\section{Results}

Sex-related transcriptome differences are driven by genes escaping XCI in the placenta. We recruited nulliparous women to a study that involved serial antenatal blood sampling, blinded ultrasound scans, and postpartum placental sampling and followed 4,212 participants from early pregnancy through birth (Table 1) (13). We compared the placental transcriptome profile of 67 male and 64 female healthy placentas using RNA sequencing (RNA-seq). We collected about $100 \mathrm{M}$ single-end 125 base reads from each subject (Supplemental Table 1; supplemental material available online with this article; https://doi.org/10.1172/jci. insight.120723DS1). A multidimensional scaling plot demonstrated profound clustering of the placental transcriptome by fetal sex (Figure 1A). After exclusion of Y chromosome genes, we observed a highly significant enrichment of differentially expressed genes on the $\mathrm{X}$ chromosome over the autosomes $(P=1.8 \times$ $10^{-42}$; Figure $1 \mathrm{~B}$ and Supplemental Table 2). Using the previously described threshold of a $10 \%$ difference in male/female transcript abundance (plus a $P<0.01$ threshold, see Methods for details) $(14,15)$, we identified $60 \mathrm{X}$ chromosome genes with sex-biased expression in the placenta (Supplemental Table 3): 47 were female-biased and 13 were male-biased. We analyzed the publicly available RNA-seq dataset from 19 other human tissues from the Genotype-Tissue Expression (GTEx) project $(16,17)$. The average number of X chromosome genes with female-biased expression was 27 (range 12-37, Supplemental Table 3), and 22 of the $\mathrm{X}$ chromosome genes exhibiting female-biased expression in the placenta did not exhibit female-biased expression in any of these other tissues (Figure 2 and Supplemental Table 4) (18).

Female-biased expression of $\mathrm{X}$ chromosome genes is commonly due to escape from X-chromosome inactivation (XCI) (19). Of the 47 female-biased genes in the placenta, only 19 had previously reported to escape from XCI $(14,15)$, and the other 28 were classified either as inactivated, variable, or unknown (Figure 2C and Supplemental Table 3). Unlike in the mouse, XCI in the human placenta is not parent-of-origin specific (20), and direct assessment of biallelic expression of X chromosome genes is not possible using analysis of mRNA from whole tissue biopsies. However, identifying genes that escape $\mathrm{XCI}$ is also informed by analysis of DNA methylation (19); escaped genes do not exhibit sex-related differences in promoter methylation, whereas the promoter regions of genes subject to XCI are generally hypermethylated in females. Therefore, we compared promoter methylation in healthy male and female placental samples using whole genome oxidative bisulphite DNA-seq (Figure 3A). Both the known and potentially novel placenta-specific escaped genes had similar levels of promoter methylation in male and female tissues, whereas genes without sex-biased expression demonstrated promoter hypermethylation in females. An analogous pattern was also observed in male-biased X chromosome genes, which exhibited promoter hypermethylation in females. We replicated these results in a further group of male and female samples using SureSelectXT Methyl-Seq (Agilent) (Figure 3, B and C, and Supplemental Tables 5 and 6). These findings indicated that the placenta had a unique profile of genes escaping XCI compared with other human tissues.

Maternal serum levels of DiAcSpm differ by fetal sex and pregnancy complications. We next analyzed metabolomic data obtained using nontargeted ultra-high-performance liquid chromatography and tandem mass spectrometry (MS/MS) of serial serum samples collected at 12, 20, 28, and 36 weeks of gestational age (wkGA) from a random sample of healthy pregnant women from our cohort $(n=279)$ and compared the results in relation to fetal sex. The metabolomic profile quantified 837 metabolites of known structural identity. We performed mixed effects regression analysis of metabolite levels across the 12, 20, and 28 wkGA samples, a potential indicator of fetoplacental synthesis. We included fetal sex interactions with gestational age in the models to identify differences between the metabolite levels in relation to fetal sex (Table 2). We then validated candidate metabolites that appeared to vary by fetal sex using serum samples from the same women obtained at $36 \mathrm{wkGA}$. This approach identified 2 circulating metabolites with different maternal serum concentration according to the sex of the fetus: 16-OH-dehydroepiandrostenedione sulfate (DHEAS) was lower when the fetus was female, whereas N1,N12-diacetylspermine (DiAcSpm) was higher when the fetus was female (Figure 4). In both cases, the sex-related difference in maternal serum concentrations was consistent with increased mRNA levels of metabolic enzymes in the female placenta (Supplemental Table 3). Lower DHEAS in women pregnant with a girl was consistent with higher expression of steroid sulfatase $(S T S)$ in the female placenta. Higher DiAcSpm in women pregnant with a girl was consistent with higher placental expression of spermine synthase (SMS) in the female placenta. Both STS and SMS are X chromosome genes, and in the placenta, both escape from XCI (Supplemental Table 6). 
Table 1. Characteristics of the participants included in the analysis of metabolites at 36 weeks of gestational age by outcome status, and all women included in the Pregnancy Outcome Prediction study

\begin{tabular}{|c|c|c|c|c|}
\hline Characteristic & $\begin{array}{c}\text { PE } \\
(n=134)\end{array}$ & $\begin{array}{c}\text { FGR } \\
(n=162)\end{array}$ & $\begin{array}{l}\text { Control } \\
(n=259)\end{array}$ & $\begin{array}{c}\text { All women } \\
(n=4,212)\end{array}$ \\
\hline \multicolumn{5}{|l|}{ Maternal characteristics } \\
\hline Age stopped FTE, years & $20(18-23)$ & $20(17-23)$ & $21(18-23)$ & $21(18-23)$ \\
\hline Missing & $2(1.5)$ & $3(1.9)$ & $1(0.4)$ & $126(3.0)$ \\
\hline Height, cm & $165(160-168)$ & 166 (161-170) & 165 (161-169) & 165 (161-169) \\
\hline \multicolumn{5}{|l|}{ Deprivation quartile } \\
\hline 1 (lowest) & $36(27)$ & $42(26)$ & $57(22)$ & $1018(24)$ \\
\hline 2 & $31(23)$ & $29(18)$ & $74(29)$ & $1002(24)$ \\
\hline 3 & $33(25)$ & $43(27)$ & $56(22)$ & $1018(24)$ \\
\hline 4 (highest) & $29(22)$ & $38(23)$ & $62(24)$ & $1002(24)$ \\
\hline European descent & 129 (96) & 154 (95) & 245 (95) & 3,900 (93) \\
\hline Missing & $1(0.8)$ & $1(0.6)$ & $5(1.9)$ & $72(1.7)$ \\
\hline Married & $89(66)$ & $97(60)$ & $188(73)$ & $2,863(68)$ \\
\hline Smoker & $2(1.5)$ & $25(15)$ & $9(3.5)$ & $211(5.0)$ \\
\hline Any alcohol consumption & $6(4.5)$ & $8(4.9)$ & $9(3.5)$ & $190(4.5)$ \\
\hline Missing & $0(0.0)$ & $0(0.0)$ & $0(0.0)$ & $2(<0.1)$ \\
\hline $\mathrm{BMI}, \mathrm{kg} / \mathrm{m}^{2}$ & $26(23-31)$ & $24(23-28)$ & $24(22-27)$ & $24(22-27)$ \\
\hline Missing & $0(0.0)$ & $0(0.0)$ & $0(0.0)$ & $7(0.2)$ \\
\hline Type 1 or type 2 DM & $1(0.8)$ & $1(0.6)$ & $0(0.0)$ & $16(0.4)$ \\
\hline Chronic hypertension & $27(20)$ & $7(4.3)$ & $5(1.9)$ & $220(5.2)$ \\
\hline Fetal sex: female & $57(43)$ & $83(51)$ & $131(51)$ & $2,080(49)$ \\
\hline Missing & $0(0.0)$ & $0(0.0)$ & $0(0.0)$ & $14(0.3)$ \\
\hline Induction of labor & $82(61)$ & $54(33)$ & $80(31)$ & $1,355(32)$ \\
\hline \multicolumn{5}{|l|}{ Mode of delivery } \\
\hline Spontaneous vaginal & $26(19)$ & $91(56)$ & $134(52)$ & 2,051 (49) \\
\hline Operative vaginal & $43(32)$ & $28(17)$ & $56(22)$ & $992(24)$ \\
\hline Prelabor caesarean & $17(13)$ & $24(15)$ & $19(7.3)$ & $424(10)$ \\
\hline Intrapartum caesarean & $48(36)$ & $19(12)$ & $48(19)$ & $726(17)$ \\
\hline Missing & $0(0.0)$ & $0(0.0)$ & $2(0.8)$ & $19(0.5)$ \\
\hline
\end{tabular}

Characteristics of (a) the participants included in the analysis of metabolites at 36 weeks of gestational age by outcome status and (b) all women included in the Pregnancy Outcome Prediction study. Data are expressed as median (IQR) or $n$ (\%) as appropriate. For fields where there is no category labeled "missing", data were $100 \%$ complete. Maternal age was defined as age at recruitment. All other maternal characteristics were defined by selfreport at the 20-week questionnaire, from examination of the clinical case record, or linkage to the hospital's electronic databases. Socioeconomic status was quantified using the Index of Multiple Deprivation (IMD) 2007, which is based on census data from the area of the mother's postcode. Birth weight centiles and the SGA (<10th centile) definition are based on the British 1990 population (53). Only term births are included in the PE, FGR, and control (healthy women) groups. The definitions of PE, FGR, and Control are given in the Supplemental Methods. There were 9 participants who had both PE and FGR, and these are included in both groups. PE, preeclampsia; FGR, fetal growth restriction; FTE, full-time education; DM, denotes diabetes mellitus. 
A

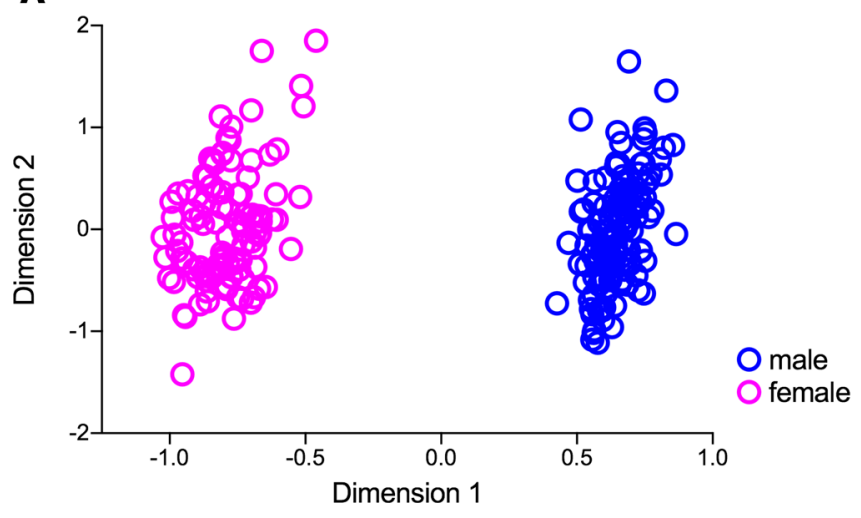

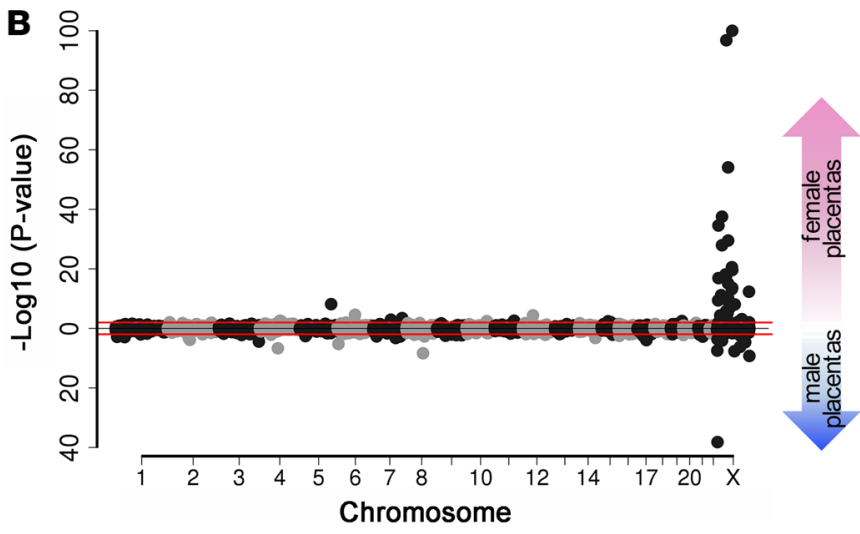

Figure 1. Analysis of the placental transcriptome by fetal sex. (A) Multidimensional scaling plot of RNA-seq data. (B) Manhattan plot of sex-biased genes on autosomes and $\mathrm{X}$ chromosome. $P$ values on the $y$ axis, Benjamini-Hochberg corrected (DESeq2). Red lines indicate adjusted $P=0.01$.

We then sought to determine whether the risk of placentally related complications of pregnancy varied according to maternal serum levels of the 2 sex-related metabolites. We compared the risk of subsequent delivery with a diagnosis of preeclampsia $(n=134)$ or subsequent delivery of a baby with FGR $(n=$ 162), with reference to 259 controls, across quintiles of DHEAS and DiAcSpm. There was no association between maternal serum DHEAS and the risk of either outcome (Supplemental Figure 1). However, the risk of preeclampsia increased $\sim 5$-fold, and the risk of FGR declined $\sim 5$-fold across quintiles of maternal serum levels of DiAcSpm (Figure 5A). Both associations were highly statistically significant $\left(P=2.5 \times 10^{-6}\right.$ and $1.1 \times 10^{-6}$, respectively) and were unaffected by adjustment for the soluble fms-like tyrosine kinase receptor 1 to placental growth factor ratio (sFLT1:P1GF, an existing placental biomarker for preeclampsia; refs. 21, 22), the sex of the fetus, and maternal characteristics (Figure 5, B-D). When the cases were stratified by phenotype, the association was strongest in the group of most severe FGR (Figure 5C). In contrast, the association did not differ comparing severe and nonsevere preeclampsia (Figure 5D). Collectively, the multiomics approach identified the polyamine pathway as differing by fetal sex and being differentially associated with the 2 major placentally related complications of human pregnancy. Therefore, we next set out to study this pathway to determine whether there was functional evidence indicating a role in sex-related differences in the human placenta.

Sex-related differences in the effects of polyamine depletion in trophoblast cells. The RNA-seq data indicated that SMS mRNA was $\sim 25 \%$ higher in the female placenta compared with the male (Figure $6 \mathrm{~A}$ and Supplemental Table 3). IHC localized the protein to the trophoblast layer of the placental villi (Figure 6B), and we confirmed higher levels of SMS protein in females using Western blot analysis of primary trophoblast cells (Figure 6C). We performed liquid chromatography-mass spectrometry (LC-MS) and studied the concentration of polyamines and their acetylated metabolites in relation to fetoplacental sex. The concentration of DiAcSpm was higher in the female placenta, consistent with the maternal serum metabolomics (Figure 6D). The functional importance of sex-related differences in placental polyamine metabolism was assessed by comparing the effect of the global polyamine synthesis inhibitor difluoromethylornithine (DFMO) on the viability of primary cultures of male versus female trophoblast cells. We found that male trophoblast cells were more sensitive to this drug than female cells (Figure 7, A and B). This demonstrated that the placenta-specific escape of SMS from XCI was associated with lower levels of SMS protein and SMS metabolites in male trophoblast cells and that male cells were more susceptible to the harmful effects of polyamine depletion.

\section{Discussion}

The key findings we report here are that differences in placental polyamine synthesis exist in male and female placentas and that this pathway is implicated in the pathophysiology of placentally related complications of human pregnancy. We used a multiomic approach to identify this pathway. We studied the placenta using RNA-seq and oxidative bisulfite DNA-seq, and we studied maternal serum using metabolomics. This approach identified 2 pathways that differed according to fetal sex. We then compared the levels of the associated metabolites in maternal serum in relation to the risk of preeclampsia and 
A

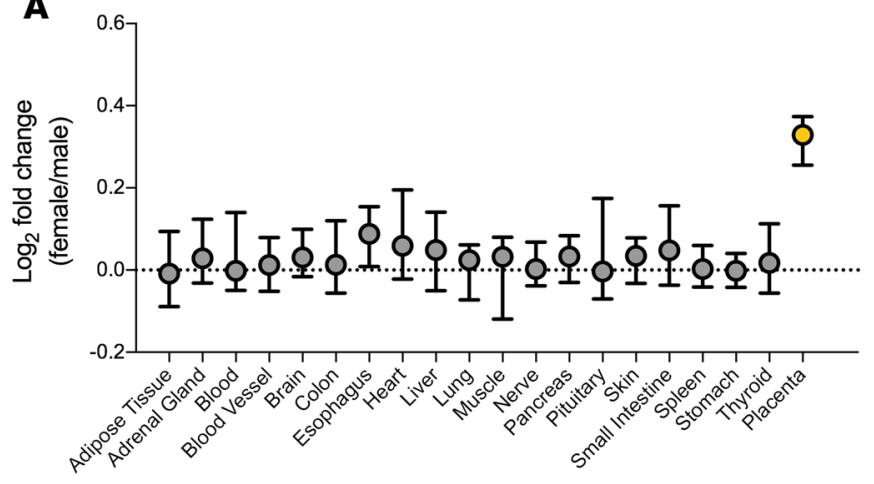

C

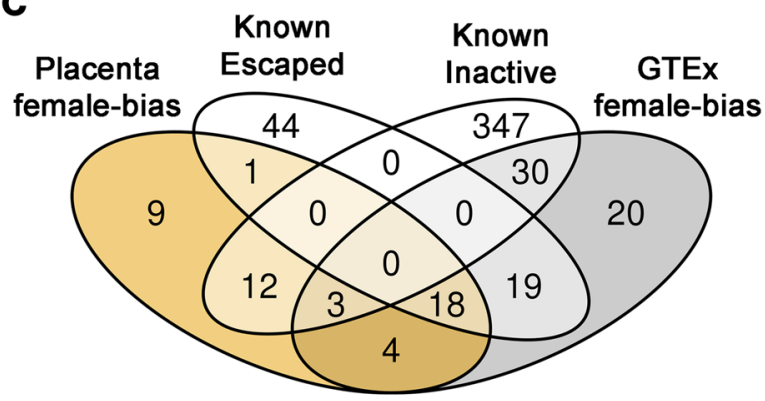

B

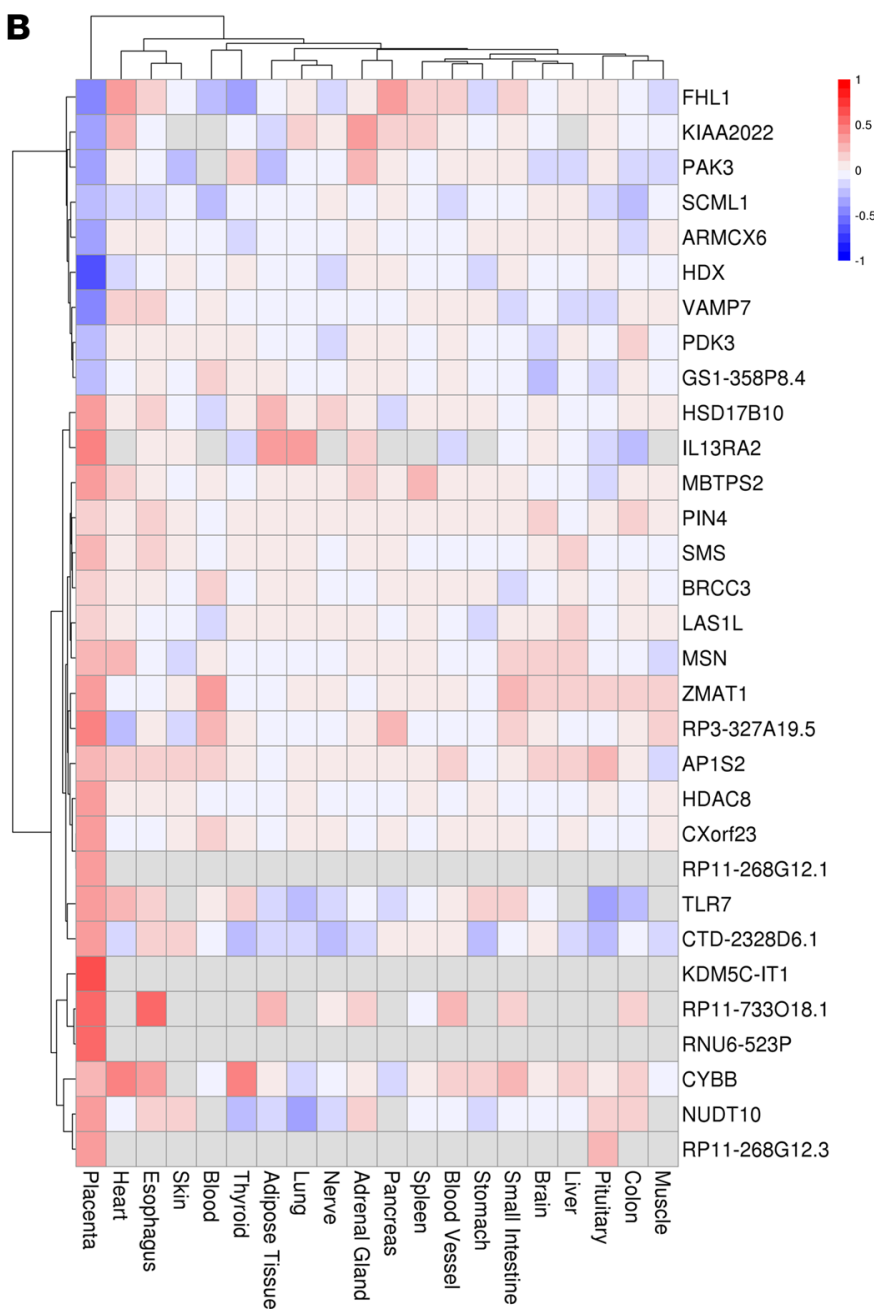

Figure 2. Placental enrichment of genes escaped from X-chromosome inactivation. (A) Transcript abundance differences in placenta and 19 other tissues (GTEX) of the 22 genes with placental female-biased expression. Points, median; bars, interquartile range (IQR). (B) Heatmap of expression ( $\log _{2}$ fold change) of previously unrecognized sex-biased X chromosome genes in placenta and 19 GTEx tissues. Red, female-biased ( $n=22$ ); blue, male-biased $(n=9)$; gray, low expression (read-count $<10$ ). (C) Venn diagram of overlap between $X$ chromosome genes with female-biased expression in placenta (placenta, female-bias) and in the 19 GTEx tissues (GTEx, female-bias), and genes previously reported to escape (known escaped) or to be subjected (known inactive) to X-inactivation.

fetal growth restriction. A single metabolite, DiAcSpm, was found to differ both in relation to fetal sex and the risk of placentally related complications of pregnancy. We then performed targeted experiments using primary culture of human trophoblast cells, which confirmed sex-related differences in the cellular effects of pharmacological interruption of the pathway. We conclude that placental sex is associated with differences in polyamine metabolism and that this variation may contribute to the known associations between fetoplacental sex and the "great obstetrical syndromes" (1).

Preeclampsia and FGR share a number of associations with biomarkers of placental function, including low first trimester of pregnancy-associated plasma protein A (PAPP-A), high second trimester $\alpha$-fetoprotein (AFP) in maternal serum (23), and high resistance patterns of uterine artery Doppler flow velocimetry $(24,25)$. However, DiAcSpm is the first circulating maternal factor to our knowledge to demonstrate opposite associations with the 2 conditions. In the present analysis, 2 serum metabolites were identified from the 837 known metabolites included in the assay. The basis for their selection was very strong statistical evidence that they varied by sex. The probability that, in the absence of association between DiAcSpm and the 2 complications (preeclampsia and FGR), we would have produced results suggesting an association this strong or stronger was in the region of 1 in 1,000,000 for both outcomes, far below the conventional 1 in 20 threshold for statistical significance. Moreover, the 5-fold 
A

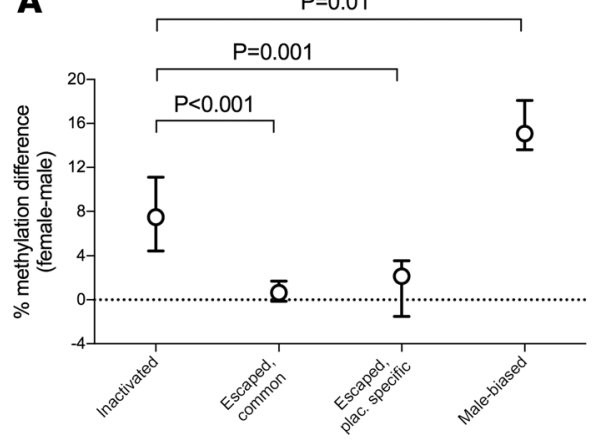

B

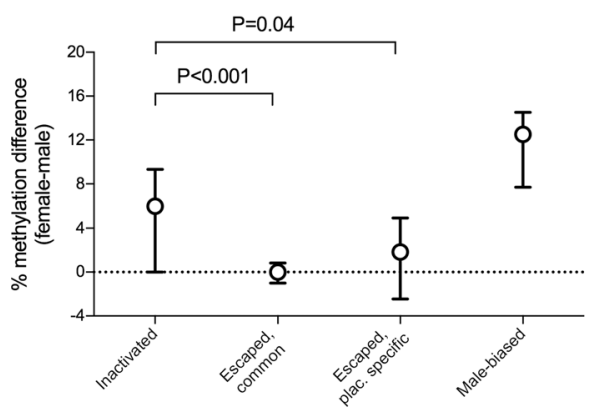

C

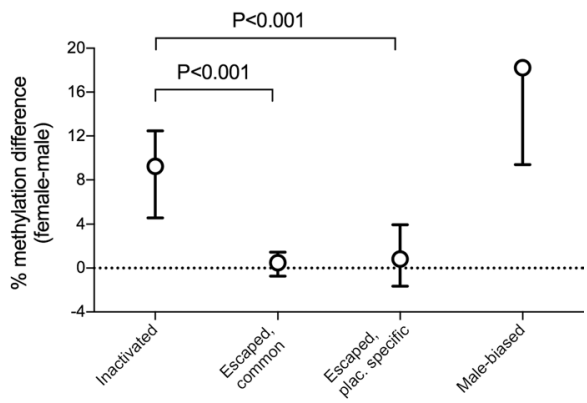

Figure 3. Promoter methylation analysis of $\mathbf{X}$ chromosome genes in male and female placentas. (A) Promoter methylation differences in male $(n=2)$ and female $(n=2)$ term placentas using WGoxBS profiles. (B and C) Technical and biological validation experiments performed using an in-solution methodology and bisufite conversion (SureSelectXT Methyl-Seq, Agilent) in (B) 2 placental samples ( $n=1$ male and $n=1$ female) previously analyzed by WGoxBS and $(\mathbf{C})$ a different set of male $(n=3)$ and female $(n=3)$ term placentas. The graphs show X chromosome genes subject to (inactivated) or escaped from $X$-chromosome inactivation, along with male-biased expression. Escaped genes uniquely identified in placenta (escaped, plac. specific) are drawn separately from those identified in at least one of 19 GTEx tissues (escaped, common). Points, median; bars, IQR. $P$ values in the graphs: Mann-Whitney $U$ test (each group vs. inactivated). Overall $P=0.0001$ (A), $P=0.0007$ (B), and $P=0.0001$ (C) obtained with Kruskal-Wallis rank test.

variation in the risk of each outcome comparing women with the lowest $20 \%$ of serum levels with women with the highest $20 \%$ of serum levels reflects a clinically important and biologically meaningful difference in risk. Our previous studies of the cohort have demonstrated that the maternal serum sFLT1:PIGF ratio is elevated at $36 \mathrm{wkGA}$ in women who subsequently experience either condition, i.e., preeclampsia or FGR without any hypertensive complications $(21,26)$. The ratio is thought to reflect placental oxidative stress, which is considered to be a feature of both conditions. The fact that the association between maternal serum levels of DiAcSpm was positive for preeclampsia but negative for FGR suggests that the ratio does not merely reflect some underlying feature common to both conditions, such as placental hypoxia. Indeed, the opposite associations suggest that the polyamine pathway may determine whether placental dysfunction results in preeclampsia, FGR, or both conditions concurrently. Unfortunately, the number of cases affected by both FGR and preeclampsia where metabolomics data were also available was too small for meaningful analysis. Similarly, there were insufficient numbers of intrauterine fetal deaths for study, and both of these are areas for future research. It also remains to be established whether any effects are local to the placenta or reflect systemic effects on the mother. A protective effect of SMS activity on FGR is supported by the phenotype of SMS deficiency in humans

Table 2. Top 10 known metabolites with different maternal serum levels by fetal sex at 12, 20, and 28 weeks of gestational age

\begin{tabular}{lccc}
\hline Biochemical & Metabolic pathway & $\begin{array}{c}\boldsymbol{P} \text { value } \\
\text { 12, 20, and 28 wkGA weeks }\end{array}$ & $\begin{array}{c}\boldsymbol{P} \text { value } \\
\mathbf{3 6} \text { wkGA weeks }\end{array}$ \\
16-OH-dehydroepiandrostendione sulfate & Steroid & $\mathbf{0 . 0 0 0 2}$ & $\mathbf{0 . 0 0 1 4}$ \\
\hline 2-stearoyl-GPI (18:0) & Lysolipid & 0.0019 & 0.8641 \\
glycoursodeoxycholate & Secondary Bile Acid Metabolism & 0.0027 & 0.1096 \\
1-stearoyl-GPI (18:0) & Lysolipid & 0.0075 & 0.7185 \\
1-linoleoyl-GPI (18:2) & Lysolipid & 0.0077 & 0.9819 \\
estriol 3-sulfate & Steroid & 0.0082 & 0.8443 \\
N1,N12-diacetylspermine & Polyamine metabolism & $\mathbf{0 . 0 0 8 4}$ \\
trimethylamine N-oxide & Phospholipid metabolism & 0.0087 & 0.0298 \\
$\alpha$-Ketobutyrate & Met, Cys, SAM, and Tau metabolism & 0.0096 & 0.5247 \\
1-Linoleoyl-GPG & Lysolipid & 0.0111 & 0.7944
\end{tabular}

Metabolites are sorted by $P$ value from the composite hypothesis test (at 12,20 , and 28 wkGA; $n=279$ ). In addition, $P$ values obtained from the analysis of male/female difference at 36 wkGA are presented (validation; $n=259$ ). Statistical significance in the 36 wkGA samples was assumed at $P<0.01$ to account for multiple testing. Fetal sex differences at any of the first 3 gestational ages (composite hypothesis) were tested using a $\chi^{2}$ test and, at 36 wkGA, using a $t$ test. Table rows corresponding to DHEAS (16-OH-dehydroepiandrostendione sulfate) and DiAcSpm (N1,N12-diacetylspermine) are marked in bold. Andicates compounds that have not been officially confirmed based on a standard. 
A

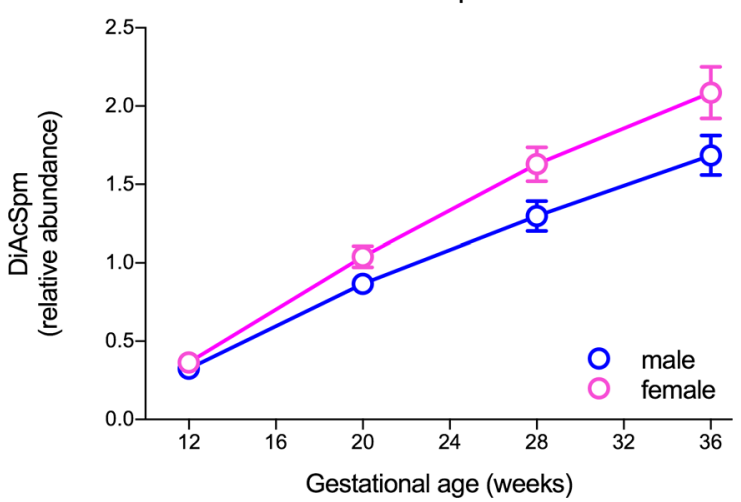

B

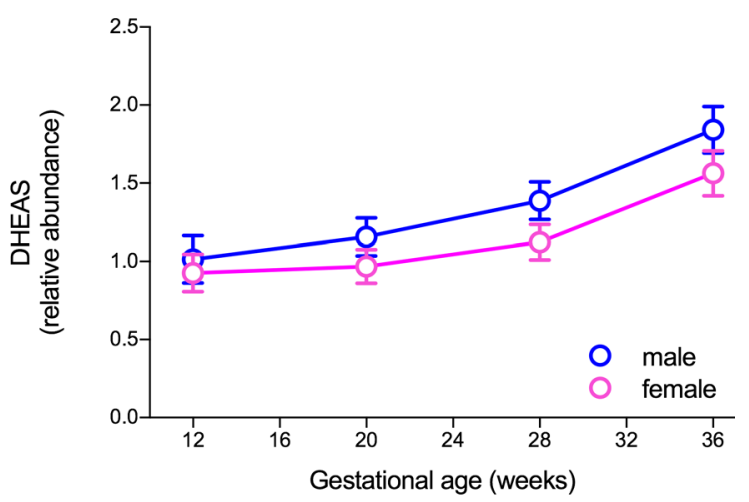

Figure 4. Maternal serum levels of DiAcSpm and DHEAS are regulated by fetal sex. Mean (points) with $95 \% \mathrm{Cl}$ (bars) at $12,20,28$, and 36 wkGA for DiAcSpm (A) and DHEAS (B) comparing normal pregnancies with male and female fetuses. Pregnancies with female fetuses were $n=146$ (12, 20 , and $28 \mathrm{wkGA}$ ) and $n=131$ (36 wkGA); pregnancies with male fetuses were $n=133(12,20$, and 28 wkGA) and $n=128$ (36 wkGA), respectively. Fetal sex differences at any of the first 3 gestational ages (composite hypothesis) were tested using a $\chi^{2}$ test and at 36 wkGA using a $t$ test. Two-sided $P$ value for the male/female difference was 0.0084 at 12, 20, and 28 wkGA and 0.0049 at 36 wkGA for DiAcSpm levels, and 0.0002 at 12, 20 , and 28 wkGA and 0.0014 at 36 wkGA for DHEAS levels.

(Snyder-Robinson syndrome), where the average birth weight (BW) of babies born at term $(27,28)$ was $\sim 500$ g less than the population average of 3,300 g (29).

The mechanisms leading to XCI are still only partially understood. The causes of tissue specificity also remain obscure. The fact that a gene might escape XCI in one tissue but not in another suggests that there might be tissue-specific regulatory factors. The majority of the genes that escaped XCI in the placenta are associated with transcriptional and translational modifications. These include zinc finger proteins (ZFX, ZRSR2, ZMAT1) and histone deacetylases (KDM5C, KDM6A, HDAC8), which regulate gene expression, as well as eukaryotic initiation factors (EIF2S3, EIFAX) and ribosomal proteins (RPS4X), which mediate protein translation. This suggests that genes that escape XCI may drive placental sexspecific responses under basal conditions or in response to environmental stimuli. However, further studies will have to address why, for example, SMS escapes XCI in the placenta but not in other tissues. Interestingly, we have recently shown sex-related differences in autosomal genes, where differential methylation of CUB And Sushi Multiple Domains 1 (CSMD1) was associated with a placenta-specific transcript and a placenta-specific sex-related difference in mRNA levels (30). Better understanding of the determinants of sex-related differences in the placenta might shed further light on key pathways involved in the pathophysiology of disease.

The current findings can also be interpreted in the light of current theories in evolutionary biology. Trivers and Willard postulated that there is a survival advantage to investment of resources in female over male offspring when resources are constrained in pregnancy (31). Our findings could be interpreted in light of this hypothesis - specifically, that placental escape of the SMS gene from XCI may enhance polyamine metabolism and confer a survival advantage to female fetuses developing with suboptimal placental function, given our discovery of a protective effect of higher DiAcSpm levels on FGR. The finding of associations in opposite directions between DiAcSpm and preeclampsia (positive association) and DiAcSpm and FGR (negative association) is particularly interesting. The 2 conditions share many common features, but it is currently unclear why placental dysfunction can lead to preeclampsia without FGR or FGR without preeclampsia. The current data indicate that placental polyamine metabolism may have a role. Haig (32) hypothesized that preeclampsia may be an adaptive response that allows the fetus to increase its share of resources by inducing maternal hypertension. This hypothesis would predict the existence of factors that increase the risk of preeclampsia but decrease the risk of FGR, and maternal serum DiAcSpm demonstrated exactly that pattern of association.

Polyamines (putrescine, spermidine, and spermine) are involved in multiple key processes implicated in many disease states, including stem cell function, autophagy, tumor suppression, immunoregulation, and both cardio- and neuro-protection (reviewed in ref. 33). Polyamines are synthesized by cells from amino acid precursors, but other sources include dietary intake and the intestinal microbiota. Dietary 
A

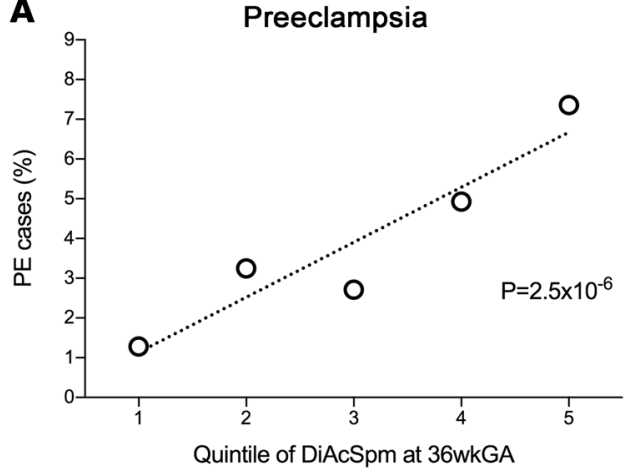

Fetal Growth Restriction

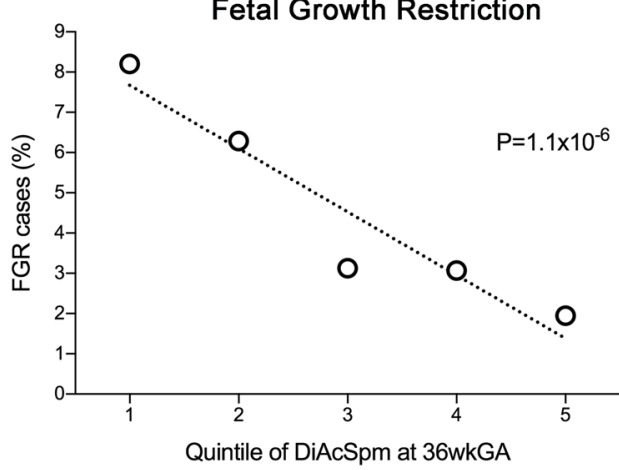

B

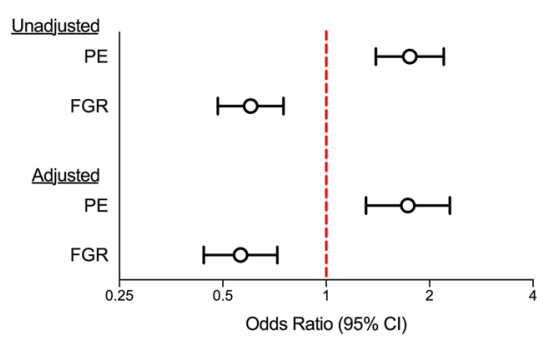

C

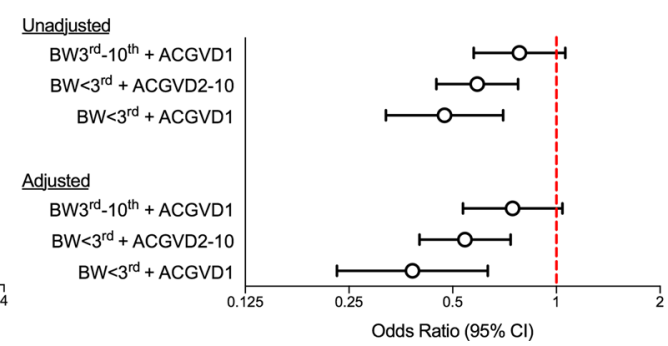

D

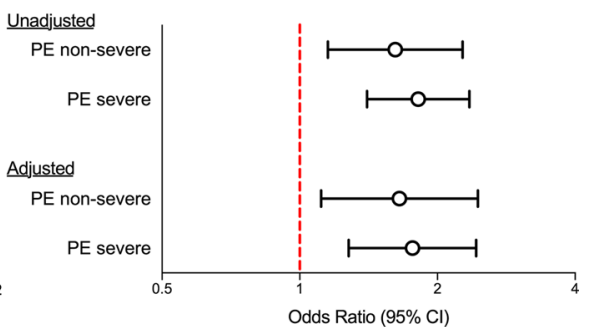

Figure 5. Maternal serum levels of DiAcSpm are regulated by pregnancy complications. (A) Proportion of cases with preeclampsia (including all severe cases and nonsevere nonsuperimposed cases as per ACOC 2013 definition; $n=134$ ) or FGR (defined as customized birth weight [BW] centile [<3rd] or the combination of customized BW centile [<10th] and abdominal circumference growth velocity [ACGV] in the lowest decile [ACGVD1]; $n=162$ ) by quintile of maternal serum DiAcSpm at 36 wkGA. $P$ values for linear trend between the quintile and log-odds of each outcome (logistic regression) are reported. (B-D) Logistic regression modeling of the association between DiAcSpm (as a continuous variable, odds ratios [ORs] expressed for a 1 SD difference) at 36 wkGA and the risk of (B) preeclampsia (PE) and FGR, as defined in A (2-sided $P<0.001$ for all ORs); (C) FGR stratified by phenotype, defined as the combination of (a) customized BW centile between the 3rd and the 10th and ACCVD1, (b) customized BW centile [ $<3 \mathrm{rd}$ ] and ACGV deciles between the 2nd and 10th (ACGVD2-10), (c) customized BW centile [ $<3$ rd] and ACGVD1 ( $n=52, n=79$, and $n=31$, respectively; 2 -sided unadjusted $P=0.11, P<0.001$, and $P<0.001$, respectively; 2 -sided adjusted $P=0.086, P<0.001$, and $P<0.001$, respectively); (D) PE stratified to nonsevere and severe cases $(n=42$ and $n=92$, respectively; 2 -sided unadjusted $P=0.005$ and $P<0.001$, respectively; 2 -sided adjusted $P=0.013$ and $P=0.001$, respectively). Reported ORs are unadjusted and adjusted for sFLT1:PIGF at 36 wkGA, fetal sex, maternal age, height, BMI, ethnicity, and smoking status.

supplementation of mice with spermidine increased longevity (34), and the potential benefits of dietary supplementation with polyamines is an area of intense interest in multiple pathological conditions. Interestingly, the human placenta had the highest ratio of SMS to spermidine synthase activity when compared across a range of human tissues (35). However, we provide the first evidence to our knowledge that the placenta exhibits a unique sex-dependent difference in polyamine metabolism, which is associated with placental-specific escape from XCI by $S M S$. Given our findings that maternal serum levels of polyamine metabolites differ by fetal sex and strongly differentially associate with the great obstetrical syndromes, understanding the effects of polyamine metabolism on placental function is an important area for future research. These associations could be of practical significance through the potential for dietary manipulation of polyamine metabolism.

\section{Methods}

\section{Study design}

The Pregnancy Outcome Prediction (POP) study was conducted at the Rosie Hospital (Cambridge, United Kingdom), as previously described $(13,36)$. In brief, it was a prospective cohort study of nulliparous women attending the hospital for their dating ultrasound scan between January 14, 2008, and July 31,2012 , with a viable singleton pregnancy. Women had blood taken at the booking visit $(\sim 12$ wkGA) and at 3 subsequent visits at the NIHR Cambridge Clinical Research Facility (at $\sim 20$ wkGA, 

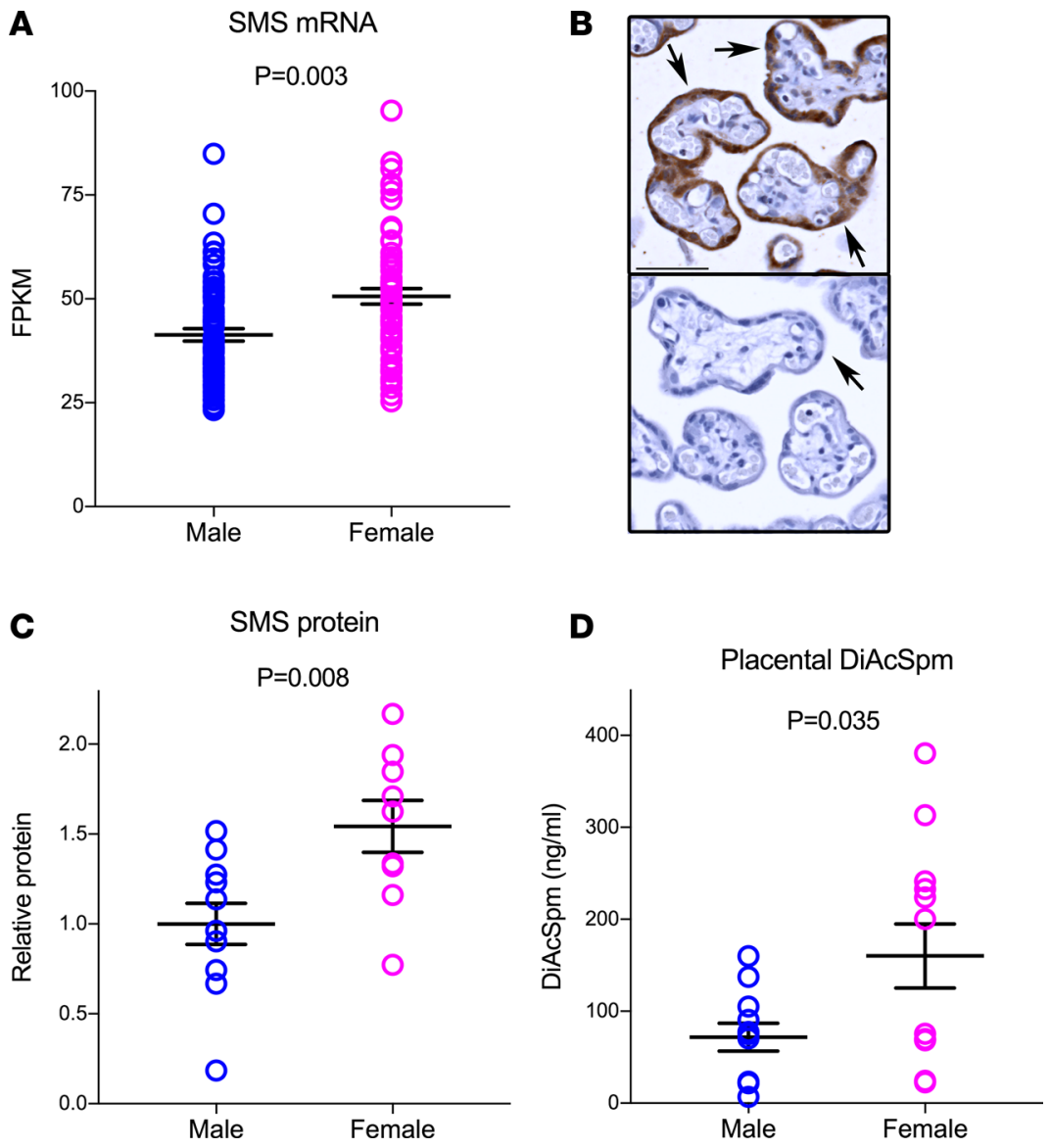

Figure 6. Higher spermine synthase expression in female placentas. (A) SMS transcript level in male $(n=67)$ and female $(n=64)$ placental tissues from healthy individuals. FPKM, fragments per kilobase of transcript per million mapped reads. (B) Immunohistochemical localization of SMS in term human placenta (top panel: rabbit anti-SMS; lower panel: rabbit IgG-control). Tissues obtained from 3 different placentas. Arrows indicate the placental trophoblast cell layer. Scale bar: $50 \mu \mathrm{m}$. (C) Western blot analysis of SMS protein in male $(n=11)$ and female $(n=9)$ primary trophoblast cells. Mean \pm SEM. Student's unpaired $t$ test. (D) LC-MS analysis of DiAcSpm in male and female placental tissues ( $n=12$ in each group). Mean \pm SEM. Student's unpaired $t$ test.

$\sim 28$ wkGA, and $\sim 36$ wkGA), when an ultrasound scan was also performed. Patients and clinicians were blinded to the results of the research ultrasound scans. The characteristics of the study cohort $(n=$ 4,212 ) by outcome status are described in Table 1.

\section{RNA-seq data processing and analysis}

Extraction of total RNA from placental biopsies and library preparation for RNA-seq are described in the Supplemental Methods.

Alignment of reads and quantification of transcript abundance. Reads were trimmed using cutadapt (37) and Trim Galore! (38) and were mapped to the GRCh37 (hg19) version of human genome reference. The so-called 2-pass (or 2-scan) alignment protocol was applied to rescue unmapped reads from the initial mapping step using TopHat2 (39), a splice-aware mapper built on top of Bowtie2 short-read aligner (40, 41). The initial and second mapped reads were merged by SAMtools (42), and the uniquely mapped reads were counted by the htseq-count of HTSeq (43) using the ENSEMBL version 75 (GENCODE 19) transcriptome definition. Transcript abundance was measured in fragments per kilobase of transcript per million mapped reads (FPKM), and the expression fold change was measured using DESeq2 Bioconductor package (44).

Identification of sex-biased genes and tissue-wide comparison. The RNA-seq data from GTEx analysis version V6p (16) were downloaded from https://gtexportal.org/home/datasets. Samples satisfying the following attributes were used: (a) mapping rate (SMMAPRT) $>0.9$; (b) exonic rate $($ SMEXNCRT) $>0.8$; and (c) mean coverage per-base $(\mathrm{SMMNCPB})>20$. Tissues having at least 20 qualifying samples of both 


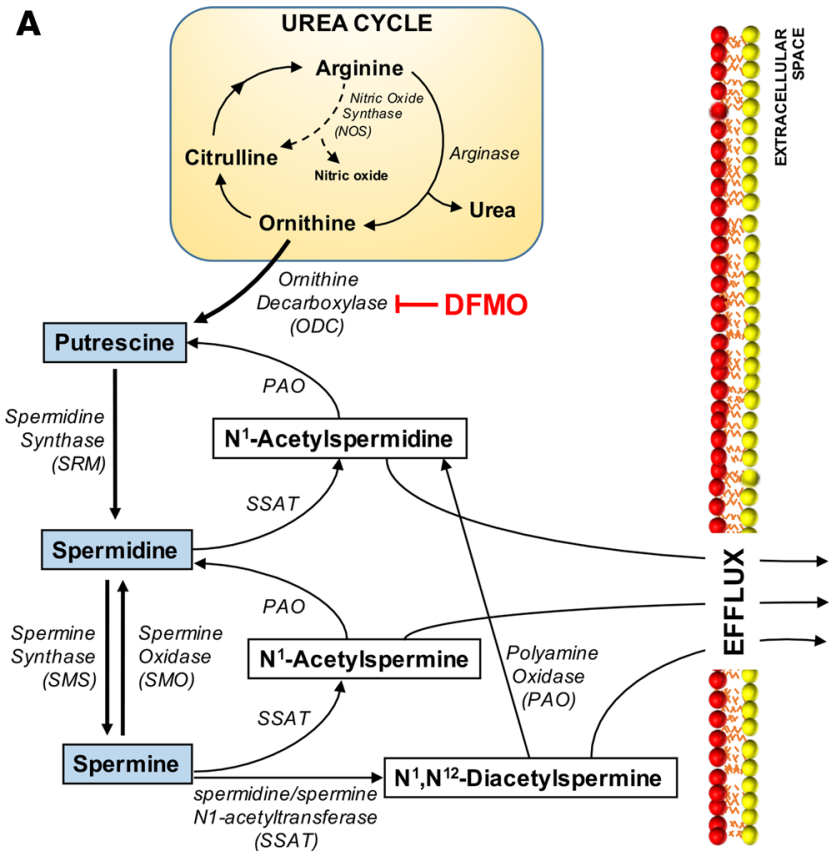

B

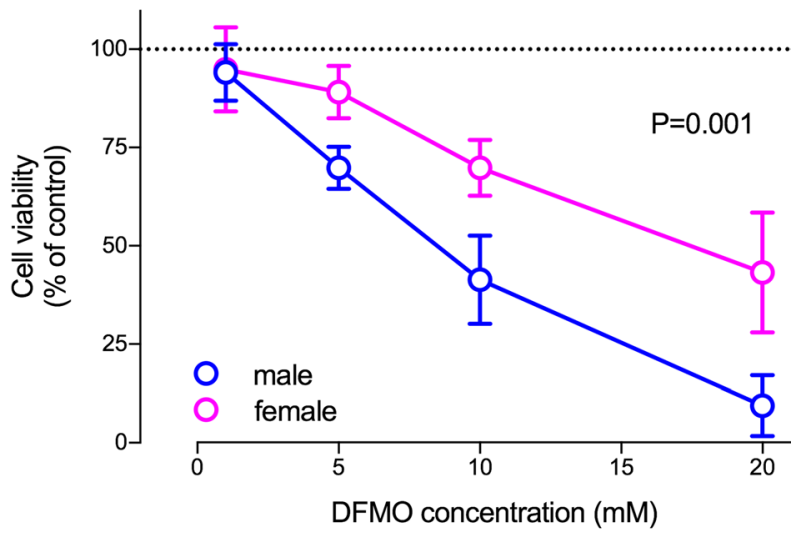

Figure 7. Polyamine depletion effects on primary trophoblast cells viability. (A) Schematic representation of the polyamine metabolic pathway. (B) Effect of the ornithine decarboxylase inhibitor, difluoromethylornithine (DFMO), on the viability of trophoblast cells isolated from male $(n=9)$ and female $(n=9)$ placentas. Points, mean; bars, $95 \% \mathrm{Cl}$. The interaction between fetal sex and the change in DFMO concentration on cell viability was estimated using a longitudinal linear mixed model with the Kenward-Roger denominator degrees of freedom method; a 2-sided exact $P$ value accounting for small-sample bias was obtained.

sexes were used, and tissue subtypes were manually selected if 2 or more were available. Additionally, sex-specific tissues were removed from the analysis: cervix, fallopian tube, ovary, prostate, testis, uterus, vagina, and breast. A total of 3,077 GTEx sample IDs selected in the final 19 tissues were analyzed in this study. Our and the GTEx data were analyzed applying the same settings in DESeq2. Chromosome Y genes were excluded from the analysis. We identified female-biased genes on chromosome X meeting the following criteria: (a) mean read-count $>10$, (b) fold change $>10 \%$ (i.e., more than $10 \%$ increase in females compared with males), and (c) Benjamini-Hochberg (45) adjusted $P<0.01$ (corrected for the number of the chromosome $\mathrm{X}$ genes from the original $P$ values). The $\mathrm{X}$ chromosome genes with sex-biased expression in placental and the 19 GTEx tissues included in this study are listed in Supplemental Table 3.

\section{Placental methylome analysis}

DNA extraction from placental biopsies is described in the Supplemental Methods.

Whole genome oxidative bisulfite sequencing (WGoxBS). Placental genomic DNA samples (4 $\mu \mathrm{g})$ from 4 healthy pregnancies ( $n=2$ /each fetal sex) were fragmented into $10 \mathrm{~Kb}$ fragments with the g-TUBE (Covaris) and concentrated using GeneJET purification columns (Thermo Fisher Scientific). Part of this fragmented DNA (1.5 $\mu \mathrm{g}$ /sample) was taken forward for oxidation + bisulfite treatment using the TrueMethyl kit (Cambridge Epigenetix). Bisulfite converted DNA (50 ng/sample) was used as input for library generation using the EpiGnome Methyl-Seq Kit (Epicentre) and EpiGnome Index PCR Primers (Epicentre). Indexed libraries were pooled in an equimolar ratio. To increase the number of uniquely sequenced reads, 2 independent libraries were generated for each individual. Multiplexed sequencing was carried out on the Illumina MiSeq and HiSeq2500 instruments with $2 \times 100,2 \times 150$ and $2 \times 125$ cycles using MiSeq Reagent Kit v3, HiSeq SBS kit v3, and HiSeq SBS kit v4, respectively. The methylation conversion rate was checked with bsExpress (https://bitbucket.org/cegx-bfx/cegx_bsexpress_docker).

In-solution target capture library preparation and sequencing. Placental genomic DNA (3.5 $\mu \mathrm{g})$ was extracted from 8 healthy pregnancies. The samples were $n=4$ /each fetal sex and included 2 samples analyzed by WGoxBS. Therefore, 2 samples represented a technical validation experiment, and 6 samples represented a biological/ technical validation experiment (using a different technique and another set of samples). The genomic DNA 
was fragmented using the Covaris S220 system and processed according to the SureSelect Methyl-Seq target enrichment protocol (Agilent). All 8 libraries were pooled and sequenced on 1 lane of the Illumina HiSeq2500 $(2 \times 125$ cycles using HiSeq SBS kit v4) and 1 lane of the Illumina HiSeq4000 $(2 \times 150$ cycles using HiSeq 3000/4000 SBS kit) following Illumina's guidelines.

Analysis of methylation sequencing data. WGoxBS and in-solution target capture data had adapter sequences removed and the quality assessed using Trim Galore! and FastQC (46), respectively. High-quality trimmed reads (i.e., base quality $\geq 20$ ) were mapped to the human genome reference (hg19) using Bismark (47). After removing reads with poor mapping quality (MAPQ $=0$ ) and duplicated reads, methylated CpGs were called using Bis-SNP (48). CpGs with at least 10× coverage were considered in the analysis. The summary statistics for the WGoxBS and in-solution target capture data processing are available in Supplemental Table 5. The methylation levels of individual CpG sites were measured by the ratio of reads with methylation (i.e., C-base reads) out of the total number of reads covering the position (i.e., sum of $\mathrm{C}$ and $\mathrm{T}$ reads). The methylation levels of promoter regions $(1,000$ $\mathrm{bp}$ upstream and 1,000 bp downstream regions of the transcript start sites) were measured if there were at least $4 \mathrm{CpGs}(\geq 10 \times)$ by weighting individual methylation levels as follows: the sum of reads with methylation out of the total number of reads over the promoter region. Sex-specific methylation differences at the promoter regions of chromosome X genes are available in Supplemental Table 6. Promoter methylation analysis of $\mathrm{X}$ chromosome genes in male and female placentas was performed comparing genes subject to XCI, escaped from XCI (divided in genes uniquely identified in placenta or identified in at least 1 of 19 GTEx tissues), and with male-biased expression. Statistical analysis was performed using Mann-Whitney $U$ tests and Kruskal-Wallis rank tests.

\section{Primary human trophoblast cell culture, Western blotting of SMS, and DFMO treatments} Human placental tissues were collected from healthy women with normal-term pregnancies and scheduled for delivery by elective cesarean section following written informed consent. Primary trophoblast cells were isolated as described in the Supplemental Methods.

Cells were harvested for Western blot analysis of SMS at 90 hours, after differentiation into syncytiotrophoblasts (Supplemental Methods). Membranes were probed with rabbit anti-SMS primary antibody $(0.5 \mu \mathrm{g} / \mathrm{ml}$, Abcam, ab156879) and peroxidase-conjugated goat anti-rabbit secondary antibody (1:2000, Cell Signaling Technology, 7074), followed by $\beta$-actin $(0.1 \mu \mathrm{g} / \mathrm{ml}$, Abcam, ab8229). Target protein level was normalized to $\beta$-actin (Image J software), and the mean density of bands from male samples were assigned an arbitrary value of 1 . Membranes were also stained for total protein using Amido Black Stain (Sigma). The results did not differ when corrected for $\beta$-actin or total protein staining (data not shown).

To analyze the effects of polyamine depletion on cell viability, after 66 hours in culture, trophoblast cells were incubated for 24 hours in the presence of DFMO $(1-20 \mathrm{mM})$ or vehicle $\left(\mathrm{H}_{2} \mathrm{O}\right)$ in media with reduced serum (1\% FBS) and $1 \mathrm{mM}$ aminoguanidine to inhibit serum amine oxidase activity (49). The viability of trophoblast cells was determined by their ability to metabolize 4,5-dimethylthiazol-2-yl-2,5-diphenyltetrazolium bromide (MTT, MilliporeSigma). Briefly, cells were incubated with $1 \mathrm{mg} / \mathrm{ml}$ of MTT reconstituted in PBS for 4 hours at $37^{\circ} \mathrm{C}$, lysed with $10 \%$ SDS, and absorbance read at $570 \mathrm{~nm}$.

\section{SMS IHC in human term placenta}

IHC was performed on formalin-fixed, paraffin-embedded placental sections as described in the Supplemental Methods, using rabbit anti-SMS (1:20 dilution) or anti-rabbit IgG (1:20 dilution, Abcam, ab172730), followed by biotin-conjugated anti rabbit secondary antibodies (1:200, Dako, E0432) and Vectastain ABC kit (Vector Labs, PK-6100).

\section{LC-MS}

LC-MS was performed on placental biopsies lysed in PBS and 10\% of spermidine-(butyl-d8) trihydrochloride internal standard (Supplemental Methods). Chromatographic separation was achieved using an ACE Excel 2 C18-PFP LC-column with a Shimadzu UPLC system (Shimadzu UK Limited). MS detection was performed on an Exactive orbitrap mass spectrometer (Thermo Fisher Scientific) operating in positive ion mode. 


\section{Serum metabolomic profiling}

Measurements and analyses. Serum metabolites were measured at Metabolon Inc. by nontargeted ultrahigh-performance LC-MS and MS/MS (50), resulting in the identification of 837 compounds of known structural identity. Peaks were quantified using AUC of primary MS ions. Samples were run in batches of 36 , and all batches contained the same proportions of cases and controls. All samples from a given woman were run in the same batch. Missing values were assumed to be the result of falling below the detection sensitivity and, thus, were imputed with the minimum detection value based on each metabolite. To adjust for instrument batch effects for each run day, the raw ion counts for each metabolite were divided by the median value for the run day. Hence, metabolites were expressed as multiples of the median, and we called this relative abundance in Figure 4.

A case-cohort design was applied (see below). Sex differences in the metabolomic profiles were assessed in a random subcohort, excluding cases $(n=279)$. We fitted longitudinal linear mixed models to identify metabolites that changed in the maternal circulation with advancing gestational age up to 28 wkGA differentially by fetal sex. Scaled imputed metabolite values (multiples of the median) were log-transformed before the analysis. We chose the top 10 metabolites differing by fetal sex with the lowest $P$ values from the composite $\chi^{2}$ test (2-sided). We then validated the result using the metabolite level at 36 wkGA from the same women by fitting a regression model between the metabolite and fetal sex. We selected the metabolites with validation $P<0.01$ for further analysis (Table 2). The distributions of the selected metabolites (DiAcSpm and DHEAS) were examined in noncases, and the best transformations were applied. Quintiles of DiAcSpm and DHEAS were calculated for all women at $36 \mathrm{wkGA}$, based on the distribution in the noncases. The association between the outcomes and the quintile of each metabolite was analyzed using logistic regression. The quintile was included in the model (a) as a categorical and (b) as a continuous exposure. All associations between the metabolite and log-odds of the outcome were approximately linear, and a 2 -sided $P$ value for linear trend was reported. To obtain the proportion of cases, the noncases were weighted by the inverse of the random subcohort sampling fraction $(4,177 / 325=12.85)$. Next, both metabolites were analyzed as continuous variables. The transformed variables were turned into $\mathrm{Z}$ scores. Logistic regression models were fitted between both metabolites at $36 \mathrm{wkGA}$ and the outcome, with and without adjustments for maternal and fetal characteristics and the sFLT1:P1GF ratio (Supplemental Methods). Similar analyses were performed on maternal serum levels of spermidine (see Supplemental Figure 2, showing no sex or disease associations with spermidine levels), while spermine was not included in the metabolomic panel.

Description of the case-cohort design used in the metabolite analysis. In total, 4,212 women completed the POP study. After excluding miscarriages, fetal deaths prior to 23 weeks and terminations $(n=29)$, and women who did not have any blood samples for analysis $(n=6), 4,177$ women remained in the cohort.

Ten groups of cases were defined, but only the preeclampsia and FGR cases born at term were included in the present study. The groups were (a) preeclampsia with preterm delivery; (b) customized BW centile $(<10$ th) (51) with preterm delivery; (c) gestational diabetes requiring drug treatment; (d) gestational diabetes requiring diet treatment; (e) spontaneous preterm delivery; (f) severe, nonsuperimposed preeclampsia, term delivery; (g) customized BW centile (<10th) with abdominal circumference growth velocity (ACGV) in the lowest decile between 20 and 36 wkGA (ACGVD1) (52); (h) severe, superimposed preeclampsia, term delivery; (i) nonsevere, nonsuperimposed preeclampsia, term delivery; and (j) customized BW centile $(<3 \mathrm{rd})$ delivered at term. In total, 644 cases belonged to 1 or more of these groups. For the present study, preeclampsia was defined by combining groups $\mathrm{f}, \mathrm{h}$, and $\mathrm{i}$ (any severe preeclampsia or nonsevere nonsuperimposed preeclampsia), as per the ACOG 2013 definition (21), and FGR was defined by combining groups g and $\mathrm{j}$ (customized BW centile $[<3 \mathrm{rd}]$ or the combination of customized BW centile $[<10 \mathrm{th}]$ and ACGVD1). Preterm births were excluded. A subcohort of 325 women was randomly selected in the cohort, and 46 of these women developed 1 or more of the 10 outcomes. The remaining 279 healthy women were used in the analysis assessing the changes in metabolites levels between $12 \mathrm{wkGA}$ and $28 \mathrm{wkGA}$. The analysis of preeclampsia and FGR was performed among women who had the metabolite measurement available at $36 \mathrm{wkGA}$ and gave birth at term. The analysis of the outcomes included 134 cases of preeclampsia, 162 cases of FGR ( 9 of these had also preeclampsia), and a shared comparison group of 259 healthy women. In a further analysis, we stratified FGR by phenotype, defined as the combination of (a) customized BW centile between the 3rd and the 10th and ACGVD1, (b) customized BW centile ( $<3 \mathrm{rd}$ ) and ACGV deciles between the 2nd and 10th (ACGVD2-10), and (c) customized BW centile $[<3 \mathrm{rd}]$ and ACGVD1 ( $n=52, n=79$ and $n=31$, respectively). Preeclampsia was stratified to nonsevere and severe cases ( $n=42$ and $n=92$, respectively). 


\section{Software and data availability}

The software used in this study is listed in Supplemental Table 7. All the computational analyses were conducted using the Linux clusters at the University of Cambridge High Performance Computing Service and the Linux workstations of School of Biological Sciences computing service. The data sets generated during the current study have been deposited in the EGA repository (https://www.ebi.ac.uk/ega/) under ID codes DNA methylation data, EGAD00001003136; RNA-seq data, EGAD00001003457.

\section{Statistics}

Statistical analyses were performed using Stata 14.2 (StataCorp). All $P$ values were 2-tailed, and statistical significance was assumed at $P<0.05$, unless otherwise specified. Specific statistical methods are further described in details in the relevant sections of Methods.

\section{Study approval}

Ethical approval was given by the Cambridgeshire 2 Research Ethics Committee (reference number 07/ H0308/163), and all participants provided written informed consent.

\section{Author contributions}

RNA-seq was contributed by FG and JD. Bioinformatics were contributed by SG, JD, and MDJ. Methylation experiments were contributed by MDJ. Statistics were contributed by US and AMW. Cell culture and immunohistochemistry were contributed by ILMHA. Technical assistance was contributed by EC. LC-MS experiments were contributed by BJJ and AK. Supervision was contributed by RAC, MC, DSCJ, and GCSS. Writing was contributed by GCSS. Manuscript approval was contributed by all authors.

\section{Acknowledgments}

We are grateful to the participants in the POP study; Leah Bibby, Samudra Ranawaka, Katrina Holmes, Josephine Gill, and Ryan Millar for technical assistance; Rabia Zill-e-Huma and Amr Gebril for reviewing clinical case records; an John Todd FRS for comments on the manuscript. The work was supported by NIHR Cambridge Comprehensive Biomedical Research Centre and the Medical Research Council (United Kingdom; G1100221 to GCSS and DSC-J and MRC_MC_UU_12012/4 to MC).

Address correspondence to: Gordon CS Smith: The Rosie Hospital, Robinson Way, Cambridge CB2 0SW, United Kingdom. Phone: 44.0.1223.336871; Email: gcss2@cam.ac.uk.

1. Brosens I, Pijnenborg R, Vercruysse L, Romero R. The "Great Obstetrical Syndromes" are associated with disorders of deep placentation. Am J Obstet Gynecol. 2011;204(3):193-201.

2. Barker DJ. Adult consequences of fetal growth restriction. Clin Obstet Gynecol. 2006;49(2):270-283.

3. Smith GC, Pell JP, Walsh D. Pregnancy complications and maternal risk of ischaemic heart disease: a retrospective cohort study of 129,290 births. Lancet. 2001;357(9273):2002-2006.

4. Zsengellér ZK, et al. Trophoblast mitochondrial function is impaired in preeclampsia and correlates negatively with the expression of soluble fms-like tyrosine kinase 1. Pregnancy Hypertens. 2016;6(4):313-319.

5. Edwards A, Megens A, Peek M, Wallace EM. Sexual origins of placental dysfunction. Lancet. 2000;355(9199):203-204.

6. Walker MG, Fitzgerald B, Keating S, Ray JG, Windrim R, Kingdom JC. Sex-specific basis of severe placental dysfunction leading to extreme preterm delivery. Placenta. 2012;33(7):568-571.

7. Møller H. Change in male:female ratio among newborn infants in Denmark. Lancet. 1996;348(9030):828-829.

8. Smith GC. Sex, birth weight, and the risk of stillbirth in Scotland, 1980-1996. Am J Epidemiol. 2000;151(6):614-619.

9. Vatten LJ, Skjaerven R. Offspring sex and pregnancy outcome by length of gestation. Early Hum Dev. 2004;76(1):47-54.

10. Neonatal and perinatal mortality: Country, regional and global estimates. World Health Organization. http://apps. who.int/ iris/handle/10665/43444. Accessed June 15, 2018.

11. Elsmén E, Källén K, Marsál K, Hellström-Westas L. Fetal gender and gestational-age-related incidence of pre-eclampsia. Acta Obstet Gynecol Scand. 2006;85(11):1285-1291.

12. Global Pregnancy Collaboration:, et al. Fetal sex-specific differences in gestational age at delivery in pre-eclampsia: a meta-analysis. Int J Epidemiol. 2017;46(2):632-642.

13. Gaccioli F, Lager S, Sovio U, Charnock-Jones DS, Smith GCS. The pregnancy outcome prediction (POP) study: Investigating the relationship between serial prenatal ultrasonography, biomarkers, placental phenotype and adverse pregnancy outcomes. Placenta. 2017;59:S17-S25.

14. Balaton BP, Cotton AM, Brown CJ. Derivation of consensus inactivation status for X-linked genes from genome-wide studies. Biol Sex Differ. 2015;6:35.

15. Carrel L, Park C, Tyekucheva S, Dunn J, Chiaromonte F, Makova KD. Genomic environment predicts expression patterns on 
the human inactive X chromosome. PLoS Genet. 2006;2(9):e151.

16. Melé M, et al. Human genomics. The human transcriptome across tissues and individuals. Science. 2015;348(6235):660-665.

17. Tukiainen T, et al. Landscape of X chromosome inactivation across human tissues. Nature. 2017;550(7675):244-248.

18. Deng X, Berletch JB, Nguyen DK, Disteche CM. X chromosome regulation: diverse patterns in development, tissues and disease. Nat Rev Genet. 2014;15(6):367-378.

19. Balaton BP, Brown CJ. Escape Artists of the X Chromosome. Trends Genet. 2016;32(6):348-359.

20. Moreira de Mello JC, et al. Random X inactivation and extensive mosaicism in human placenta revealed by analysis of allele-specific gene expression along the X chromosome. PLoS One. 2010;5(6):e10947.

21. Sovio U, Gaccioli F, Cook E, Hund M, Charnock-Jones DS, Smith GC. Prediction of Preeclampsia Using the Soluble fms-Like Tyrosine Kinase 1 to Placental Growth Factor Ratio: A Prospective Cohort Study of Unselected Nulliparous Women. Hypertension. 2017;69(4):731-738.

22. Zeisler H, et al. Predictive Value of the sFlt-1:P1GF Ratio in Women with Suspected Preeclampsia. N Engl J Med. 2016;374(1):13-22.

23. Smith GC, Stenhouse EJ, Crossley JA, Aitken DA, Cameron AD, Connor JM. Early pregnancy levels of pregnancy-associated plasma protein a and the risk of intrauterine growth restriction, premature birth, preeclampsia, and stillbirth. J Clin Endocrinol Metab. 2002;87(4):1762-1767.

24. Yu CK, Smith GC, Papageorghiou AT, Cacho AM, Nicolaides KH, Fetal Medicine Foundation Second Trimester Screening Group. An integrated model for the prediction of preeclampsia using maternal factors and uterine artery Doppler velocimetry in unselected low-risk women. Am J Obstet Gynecol. 2005;193(2):429-436.

25. Smith GC, Yu CK, Papageorghiou AT, Cacho AM, Nicolaides KH, Fetal Medicine Foundation Second Trimester Screening Group. Maternal uterine artery Doppler flow velocimetry and the risk of stillbirth. Obstet Gynecol. 2007;109(1):144-151.

26. Gaccioli F, Sovio U, Cook E, Hund M, Charnock-Jones DS, Smith GCS. Screening for fetal growth restriction using ultrasound and the sFLT1 to PIGF ratio in nulliparous women: a prospective cohort study [published online ahead of print June 7, 2018] Lancet Child Adolesc Health. https://doi.org/10.1016/S2352-4642(18)30129-9.

27. Becerra-Solano LE, et al. A missense mutation, p.V132G, in the X-linked spermine synthase gene (SMS) causes Snyder-Robinson syndrome. Am J Med Genet A. 2009;149A(3):328-335.

28. Albert JS, et al. Impaired osteoblast and osteoclast function characterize the osteoporosis of Snyder - Robinson syndrome. Orphanet J Rare Dis. 2015;10:27.

29. Kiserud T, et al. The World Health Organization Fetal Growth Charts: A Multinational Longitudinal Study of Ultrasound Biometric Measurements and Estimated Fetal Weight. PLoS Med. 2017;14(1):e1002220.

30. Gong S, et al. Genome-wide oxidative bisulfite sequencing identifies sex-specific methylation differences in the human placenta Epigenetics. 2018;13(3):228-239.

31. Trivers RL, Willard DE. Natural selection of parental ability to vary the sex ratio of offspring. Science. 1973;179(4068):90-92.

32. Haig D. Genetic conflicts in human pregnancy. Q Rev Biol. 1993;68(4):495-532.

33. Madeo F, Eisenberg T, Pietrocola F, Kroemer G. Spermidine in health and disease. Science. 2018;359(6374):eaan2788.

34. Eisenberg T, et al. Cardioprotection and lifespan extension by the natural polyamine spermidine. Nat Med. 2016;22(12):1428-1438.

35. Kajander EO, Kauppinen LI, Pajula RL, Karkola K, Eloranta TO. Purification and partial characterization of human polyamine synthases. Biochem J. 1989;259(3):879-886.

36. Pasupathy D, Dacey A, Cook E, Charnock-Jones DS, White IR, Smith GC. Study protocol. A prospective cohort study of unselected primiparous women: the pregnancy outcome prediction study. BMC Pregnancy Childbirth. 2008;8:51.

37. Martin JA, Wang Z. Next-generation transcriptome assembly. Nat Rev Genet. 2011;12(10):671-682.

38. Krueger F. Trim Galore! Babraham Bioinformatics. http://www.bioinformatics.babraham.ac.uk/projects/trim_galore/ Accessed June 15, 2018.

39. Kim D, Pertea G, Trapnell C, Pimentel H, Kelley R, Salzberg SL. TopHat2: accurate alignment of transcriptomes in the presence of insertions, deletions and gene fusions. Genome Biol. 2013;14(4):R36.

40. Liao Y, Smyth GK, Shi W. The Subread aligner: fast, accurate and scalable read mapping by seed-and-vote. Nucleic Acids Res. 2013;41(10):e108.

41. Langmead B, Salzberg SL. Fast gapped-read alignment with Bowtie 2. Nat Methods. 2012;9(4):357-359.

42. Li H, et al. The Sequence Alignment/Map format and SAMtools. Bioinformatics. 2009;25(16):2078-2079.

43. Anders S, Pyl PT, Huber W. HTSeq--a Python framework to work with high-throughput sequencing data. Bioinformatics. 2015;31(2):166-169.

44. Love MI, Huber W, Anders S. Moderated estimation of fold change and dispersion for RNA-seq data with DESeq2. Genome Biol. 2014;15(12):550.

45. Benjamini Y, Hochberg Y. Controlling the false discovery rate: a practical and powerful approach to multiple testing. $J R$ Stat Soc Series B Stat Methodol. 1995;57(1):289-300.

46. Simon A. FastQC: a quality control tool for high throughput sequence data. Babraham Bioinformatics. http://www.bioinformatics.babraham.ac.uk/projects/fastqc. Accessed June 15, 2018.

47. Krueger F, Andrews SR. Bismark: a flexible aligner and methylation caller for Bisulfite-Seq applications. Bioinformatics. 2011;27(11):1571-1572.

48. Liu Y, Siegmund KD, Laird PW, Berman BP. Bis-SNP: combined DNA methylation and SNP calling for Bisulfite-seq data. Genome Biol. 2012;13(7):R61.

49. Rider JE, Hacker A, Mackintosh CA, Pegg AE, Woster PM, Casero RA. Spermine and spermidine mediate protection against oxidative damage caused by hydrogen peroxide. Amino Acids. 2007;33(2):231-240.

50. Evans AM, DeHaven CD, Barrett T, Mitchell M, Milgram E. Integrated, nontargeted ultrahigh performance liquid chromatography/electrospray ionization tandem mass spectrometry platform for the identification and relative quantification of the small-molecule complement of biological systems. Anal Chem. 2009;81(16):6656-6667.

51. Gardosi J, Mongelli M, Wilcox M, Chang A. An adjustable fetal weight standard. Ultrasound Obstet Gynecol. 1995;6(3):168-174.

52. Sovio U, White IR, Dacey A, Pasupathy D, Smith GCS. Screening for fetal growth restriction with universal third trimester 
ultrasonography in nulliparous women in the Pregnancy Outcome Prediction (POP) study: a prospective cohort study. Lancet. 2015;386(10008):2089-2097.

53. Freeman JV, Cole TJ, Chinn S, Jones PR, White EM, Preece MA. Cross sectional stature and weight reference curves for the UK, 1990. Arch Dis Child. 1995;73(1):17-24. 